\title{
A case of recurrent malignant fibrous histiocytoma with marked response to combined chemotherapy with gemcitabine and carboplatin
}

\author{
Zhang Wei ${ }^{1}$, Luan Li ${ }^{2}$, Xiao-Yu Xü , Hong Wang ${ }^{4}$, Aman Wang ${ }^{5}$, Hai-Bo Zhu' ${ }^{6}$, Qian Zhang ${ }^{7}$, Ling Wang ${ }^{5}$
}

\author{
${ }^{1}$ Pharmaceutical Department, The First Affiliated Hospital of Dalian Medical \\ University, Dalian, China \\ ${ }^{2}$ Department of Chemotherapy, Jiangsu Cancer Hospital, Jiangsu, China \\ ${ }^{3}$ Department of Oncology, Weihai Wendeng Center Hospital, Weihai, China \\ ${ }^{4}$ Department of Orthopedics, The First Affiliated Hospital of Dalian Medical University, \\ Dalian, China \\ ${ }^{5}$ Department of Oncology, The First Affiliated Hospital of Dalian Medical University, \\ Dalian, China \\ ${ }^{6}$ Department of Oncology, Affiliated Hospital of Chengdu University, Chengdu, China \\ ${ }^{7}$ Dalian Medical University, Dalian, China
}

Submitted: 31 July 2013
Accepted: 27 August 2013

Arch Med Sci 2014; 10, 5: 1057-1060

DOI: 10.5114 /aoms.2014.44874

Copyright (c) 2014 Termedia \& Banach

A 60-year-old man began to complain of a mass and pain in the right thigh for 3 months. Since these symptoms steadily progressed, he visited a local hospital in Apr 2008, where he underwent a locality tumorectomy. Histologic examination of the excisional biopsy specimen showed malignant fibrous histiocytoma (MFH) characteristics. The patient refused further treatment and returned home. The patient was readmitted to "The Third People's Hospital Of Dalian" in Apr 2010, because of repeated local recurrence and severe pain over a 5-month period. Under these circumstances, the patient consented to second surgery and right hip disarticulation was performed. No adjuvant chemotherapy or irradiation was given. In Sep 2010, the patient presented with a recurrent tumor on the stump of the right lower limb and severe pain again. The patient was admitted to "Beijing Ji Shuitan Hospital". The spiral chest computed tomography scan revealed multiple variable size nodules whose bilateral lung maximum diameter was $3.4 \mathrm{~cm}$. A stump of right thigh computed tomography (CT) and magnetic resonance imaging (MRI) showed two hypodense masses, which presented inhomogeneous contrast enhancement and scatter. Bone scan failed to show bone metastases. On 16 Dec 2010, the patient underwent the third surgery for the stump lesion of the right thigh resection. Histological studies of the mass revealed moderate anaplasia, with spindle cells of uneven density in fibrous stroma, suggesting spindle cell sarcoma Broders grade III, consistent with MFH (no obvious structure of storiform). Fifteen days after the surgery, two cycles of chemotherapy were carried out with ifosfamide and doxorubicin, respectively. According to RECIST1.1 criteria, the therapeutic evaluation was defined as PD.

On 11 Feb 2011, the patient visited the First Affiliated Hospital of Dalian Medical University, with the chief complaint of chest pain, dyspnea and cough. Physical examination revealed low breath sounds over the left lung field. A chest CT scan showed a large amount of pleural

\author{
Corresponding author: \\ Ling Wang \\ Department of Oncology \\ The First Affiliated Hospital \\ of Dalian Medical University \\ 222 Zhongshan St \\ 116011 Dalian, China \\ Phone: 15541190869 \\ E-mail: whwl2003@tom.com
}



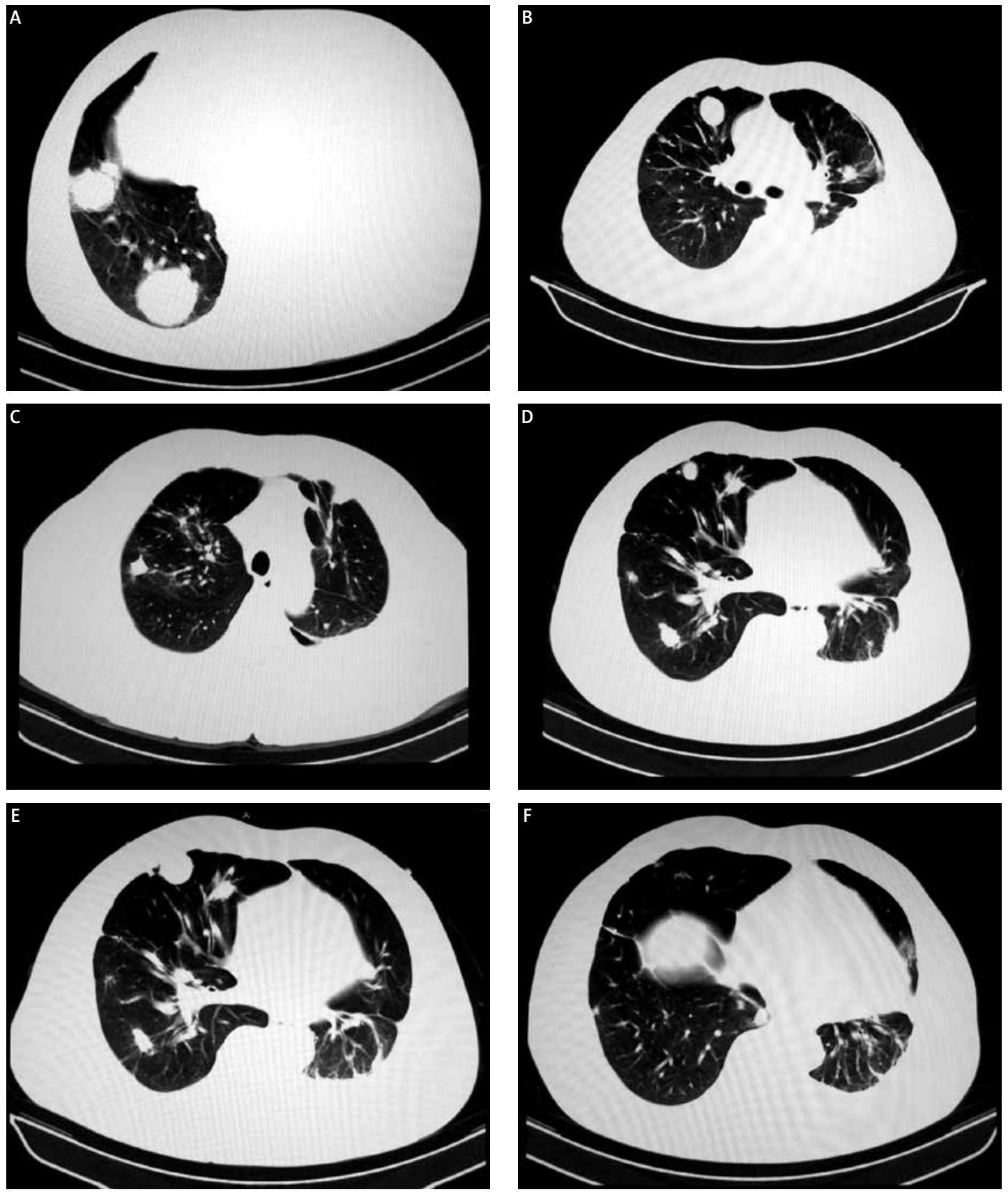

Figure 1. Patient's chest CT scan from 11 February 2011 to 27 July 2011 (A - 2011.02.11, B - 2011.3.15, C 2011.4.20, D - 2011.5.23, E - 2011.7.4, F - 2011.7.27)

effusion in the left thoracic cavity and multiple variable size nodules in the bilateral lung. A whole abdominal CT scan failed to show any metastases. From 16 Feb 2011, we gave the patient two cycles of combination chemotherapy with gemcitabine hydrochloride plus carboplatin; the first cycle of carboplatin was given by intrathoracic perfusion after thoracocentesis. The chest CT scan showed a reduction in the size and marked liquefaction necrosis of the lung metastases; according to the RECIST1.1 criteria, the therapeutic evaluation was a partial response. Another two cycles of combination chemotherapy were performed. After a total of four sequential cycles, evolution of therapeutic efficiency is progressive disease (PD). We changed the regimen to paclitaxel plus carboplatin; the therapeutic evaluation was stability of disease. The patient went home after two cycles. He died of hemoptysis at a local hospital on 16 Sep 2011 (Figure $1 \mathrm{~A}-\mathrm{F}$ ).

Soft tissue sarcomas (STS) are the most common sarcomas (approximately 76\%) [1]. They are a heterogeneous family of malignancies originating from mesenchymal tissues with different biological and clinical characteristics [2]. The bulk of evidence from all these studies suggests that 
MFH is a sarcoma of either fibroblastic or primitive mesenchymal origin, which manifests features of both fibroblastic and histiocytic differentiation [3]. So in the 2002 World Health Organization classification of soft tissue tumors, it was reestablished as undifferentiated pleomorphic sarcoma (UPS), including pleomorphic, giant cell, myxoid/ high-grade myxofibrosarcoma and inflammatory forms. The primary tumour mostly localized at the extremities (60\%), especially the thigh, and trunk (19\%); less common sites include the retroperitoneum (15\%), and the head and neck (9\%) $[1,4]$. Histologic type, local recurrence, positive microscopic margins, and the addition of adjuvant therapy in the form of either radiation or chemotherapy did not have a significant influence on the overall survival of the patients. Five-year survival for stages I, II, III, and IV are approximately $90 \%$, $70 \%, 50 \%$, and $10 \%$ to $20 \%$, respectively [1].

Almost $30 \%$ of patients treated with doxorubicin achieve an objective response, and 5\% have better long-term disease-free survival [5]. Ifosfamide is also an effective agent, with $7-38 \%$ overall objective response, and can be used in combination with doxorubicin for patients with STS with good performance status. These two drugs are used to treat the large majority of soft tissue sarcomas as first-line treatment. This combination offers a response rate of approximately 30-59\% of patients with soft tissue sarcoma. In previous phase II studies of active chemotherapy agents in sarcoma, overall survival (OS) and progression-free survival (PFS) were 13.3 vs. 11.9 and 6.1 vs. 3.9 months, respectively, in the study of doxorubicin-dacarbazine with and without ifosfamide. However, the patient with MFH in this case we report had failed with ifosfamide and doxorubicin respectively.

Other chemotherapeutic agents have also been tested in clinical trials, such as gemcitabine alone, gemcitabine and docetaxel, and gemcitabine and vinorelbine.

Gemcitabine administered as a single agent has been evaluated as second-line therapy in the treatment of soft tissue sarcoma. Many phase II trials have reported response rates of single-agent gemcitabine in patients with advanced STS ranging from $3 \%$ to $20.5 \%$. The median time to progression was 1.5 to 13 months, while median overall survival was 6 to 15 months [6-9]. It might indicate that $\mathrm{MFH}$ is sensitive to gemcitabine alone [10].

Currently, the combination of gemcitabine and docetaxel in many clinical studies in advanced soft tissue sarcomas has been found highly active.

A retrospective study by Leu et al. observed an overall response rate of $43 \%$; median time to progress (TTP) was 6.7 months and median OS was estimated at 13 months. The response rate for leiomyosarcoma (LMS) was 58\% and that for histological other pathological type was 35\% [11]. In a randomized phase II study of Maki et al. the objective response rate was $16 \%$ with the combination of gemcitabine and docetaxel. The overall response rate was $17 \%$ with LMS compared to $36 \%$ with $\mathrm{MFH}$. The median PFS and median OS were 6.2 months and 17.9 months in patients with metastatic STS, respectively [10]. This study also suggested that LMS and MFH might be more sensitive than other sarcomas to gemcitabine-docetaxel [10, 11].

It is important to recognize that gemcitabine with docetaxel is potentially associated with significant toxicity. The commonly adverse effects that were noted included thrombocytopenia, neutropenia, fatigue, and myalgias [10, 11]. Pulmonary toxicity (20\%) and refractory peripheral edema are the most common severe adverse events worth noting [10]. More than $50 \%$ of patients receiving gemcitabine and docetaxel discontinued treatment within 6 months of therapy, despite dose reductions [12].

In this case, the patient with MFH, who had failed with ifosfamide and doxorubicin, had a poor performance status. Considering that the patient cannot tolerate gemcitabine-docetaxel and gemcitabine-vinorelbine, we treated the patient with combination chemotherapy of gemcitabine hydrochloride plus carboplatin, which led to a reduction in the size and marked liquefaction necrosis of the lung metastases. According to RECIST1.1 criteria, the response to treatment was considered as a partial response. After the total of four cycles, progressive of disease was obtained. We changed the regimen to paclitaxel plus carboplatin, and the patient obtained stabilization of disease. The patient died of hemoptysis on 16 Sep 2011. The PFS and OS were 4.6 and 7 months respectively.

In the case we report, the regimen gemcitabine hydrochloride plus carboplatin is less toxic. The patient had only grade 2 hematological toxicity and nausea.

In conclusion, the regimen of combination chemotherapy with gemcitabine hydrochloride and carboplatin showed clinical benefit response to MFH and was less toxic. Obviously, the results need to be confirmed and validated in prospective studies that might clearly demonstrate a synergistic or an additive effect of gemcitabine and carboplatin.

\section{Acknowlegdments}

Zhang Wei, Luan Li and Xiao-Yu Xu contributed equally to this manuscript. 


\section{References}

1. Nedea EA, DeLaney TF. Sarcoma and skin radiation oncology. Hematol Oncol Clin N 2006; 20: 401-29.

2. Ferraresi V, Ciccarese M, Cercato MC, et al. Gemcitabine at fixed dose-rate in patients with advanced soft-tissue sarcomas: a mono-institutional phase II study. Cancer Chemother Pharmacol 2008; 63: 149-55.

3. Al-Agha OM, Igbokwe AA. Malignant fibrous histiocyto$\mathrm{ma}$ - between the past and the present. Arch Pathol Lab Med 2008; 132; 1030-5.

4. Cormier JN, Pollock RE. Soft tissue sarcomas. Ca-Cancer J Clin 2004; 54: 94-109.

5. Kanjeekal S, Chambers A, Fung MFK, Verma S, Guidlin CCOP. Systemic therapy for advanced uterine sarcoma: a systematic review of the literature. Gynecol Oncol 2005; 97: 624-37.

6. Okuno S, Ryan LM, Edmonson JH, Priebat DA, Blum RH. Phase II trial of gemcitabine in patients with advanced sarcomas (E1797): a trial of the Eastern Cooperative Oncology Group. Cancer 2003; 97: 1969-73.

7. Look KY, Sandler A, Blessing JA, Lucci JA 3rd, Rose PG; Gynecologic Oncology Group (GOG) Study. Phase II trial of gemcitabine as second-line chemotherapy of uterine leiomyosarcoma: a Gynecologic Oncology Group (GOG) Study. Gynecol Oncol 2003; 92: 644-7.

8. Hartmann JT, Oechsle K, Huober J, et al. An open label, non-comparative phase II study of gemcitabine as salvage treatment for patients with pretreated adult type soft tissue sarcoma. Invest New Drugs 2006; 24: 249-53.

9. Von Burton G, Rankin C, Zalupski MM, Mills GM, Borden EC, Karen A. Phase II trial of gemcitabine as first line chemotherapy in patients with metastatic or unresectable soft tissue sarcoma. Am J Clin Oncol 2006. 29: 59-61.

10. Maki RG, Wathen JK, Patel SR, et al. Randomized phase Il study of gemcitabine and docetaxel compared with gemcitabine alone in patients with metastatic soft tissue sarcomas: results of sarcoma alliance for research through collaboration study 002 [corrected]. J Clin Oncol 2007; 25: 2755-63.

11. Leu KM, Ostruszka LJ, Shewach D, et al. Laboratory and clinical evidence of synergistic cytotoxicity of sequential treatment with gemcitabine followed by docetaxel in the treatment of sarcoma. J Clin Oncol 2004; 22: 1706-12.

12. Maki RG. Gemcitabine and docetaxel in metastatic sarcoma: past, present, and future. Oncologist 2007; 12: 999-1006. 